\title{
Optical Low Resolution Spectroscopic Observations of T Pyx during the Early Phase of 2011 Outburst
}

\author{
A. Arai ${ }^{1,2}$, M. Isogai ${ }^{2,3}$, M. Yamanaka ${ }^{4}$, H. Akitaya ${ }^{5}$, M. Uemura ${ }^{5}$ \\ ${ }^{1}$ Center for Astronomy, University of Hyogo \\ ${ }^{2}$ Koyama Astronomical Observatory, Kyoto Sangyo University \\ ${ }^{3}$ National Astronomical Observatory of Japan \\ ${ }^{4}$ Kwasan Observatory, Kyoto University \\ ${ }^{5}$ Higashi-Hiroshima Astronomical Observatory, Hiroshima University \\ Corresponding author: arai@nhao.jp
}

\begin{abstract}
We report on the results of our low resolution spectroscopic observations during the 2011 outburst of the recurrent nova T Pyx. Our observations were performed from 0.19 days to 34 days after the eruption discovered by M. Linnolt. We found Wolf-Rayet like features in our spectrum during the initial rising phase on $t=0.19 \mathrm{~d}$. Following spectral developments are consistent with previous works. We discuss that the early phase of T Pyx is divided into three stages, a short lived WR-like stage, $\mathrm{He} / \mathrm{N}$ stage and Fe II stage.
\end{abstract}

Keywords: cataclysmic variables - optical - spectroscopy - individual: T Pyx.

\section{Introduction}

The initial rising phase of novae is a very intriguing phase in the point of view of thermonuclear runaway and development of spectral features. Generally, observations are very poor in the initial rising phase, because it is very rare that novae are discovered during their initial rising phase. Especially, spectroscopic observations lack at these very early epochs.

T Pyx is one of the recurrent novae. Its previous outbursts were observed in 1890, 1902, 1920, 1944, and 1967 (Schaefer et al. 2010). Low resolution spectroscopic observations in past outbursts were performed around their visual maximum (e.g., Adams and Joy 1920, Joy 1945, and Catchpole et al. 1969). Recurrent novae (RNe) are semi-detached binary systems consisting of a massive white dwarf with accretion material from a companion star (at typical mass transfer rate $\left.\simeq 10^{-7} \mathrm{M}_{\odot} \mathrm{yr}^{-1}\right)$. They are the prime candidates for being type Ia supernovae ( $\mathrm{SNe}$ ) progenitor. Recurrent novae are classified into three types, the U Sco-, the RS Oph- and the T Pyx-types, based on the light curves and spectroscopic features (Anupama et al. 2008). But, the light curve of $\mathrm{T}$ Pyx in the early phase is more similar to slow rising classical novae, than the U Sco- or the RS Oph-type RNe. In past outbursts, T Pyx showed a rapid rising to $V \sim 8$, followed by a pre-maximum halt and a further slow rising to the maximum light
$(V \sim 6.5)$ for $\sim 20$ days with variations of $\Delta V \sim 0.2$.

The sixth outburst of T Pyx was discovered at visual magnitude 13.0 on UT 2011 April 14.2931 (= JD $2455665.7931, t=0 \mathrm{~d}$ ) by M. Linnolt (Waagan et al. 2011). After the discovery, many observers carried out follow up observations. The study in the early spectroscopic observations and photometry revealed that T Pyx showed rare hybrid spectral classification types (Williams 1992), He/N - Fe II - He/N transition (e.g. Izzo et al. 2012, Imamura and Tanabe 2012, Surina et al. 2014). Following prompt Target of Opportunity observations were also performed in X-ray and Radio just after the discovery of the outburst (e.g., Kuulkers et al. 2011, Nordsieck and Shara 2011, Chomiuk et al. 2011). Here, we report our spectroscopic results of T Pyx from the very early phase. In this paper, we focused on the Wolf-Rayet feature in the initial rising phase on $t=0.19 \mathrm{~d}$ (2011 April 14.48) and following developments of spectral features from $t=2 \mathrm{~d}$ to $t=34 \mathrm{~d}$.

\section{Observations}

Our low dispersion optical spectroscopic observations of T Pyx were performed during 13 nights from 2011 April 14 to May 18 at two sites. One site is Koyama Astronomical Observatory (Kyoto Sangyo University). We used the Araki telescope with LOSA/F2 spectrograph (Shinnaka et al. 2013). The wavelength coverage 
is $4000-8000 \AA$ and the spectral resolution is $R \sim 580$ at $6563 \AA$. Another one is Higashi-Hiroshima Astronomical Observatory (Hiroshima University). We use the Kanata telescope with HOWPol (Kawabata et al. 2008). The wavelength coverage is $4200-9000 \AA$ and the spectral resolution of $R \sim 400$ at $6563 \AA$. The V-band light curve and epochs of our spectroscopic observations are shown in the panel (a) of Figure 1.

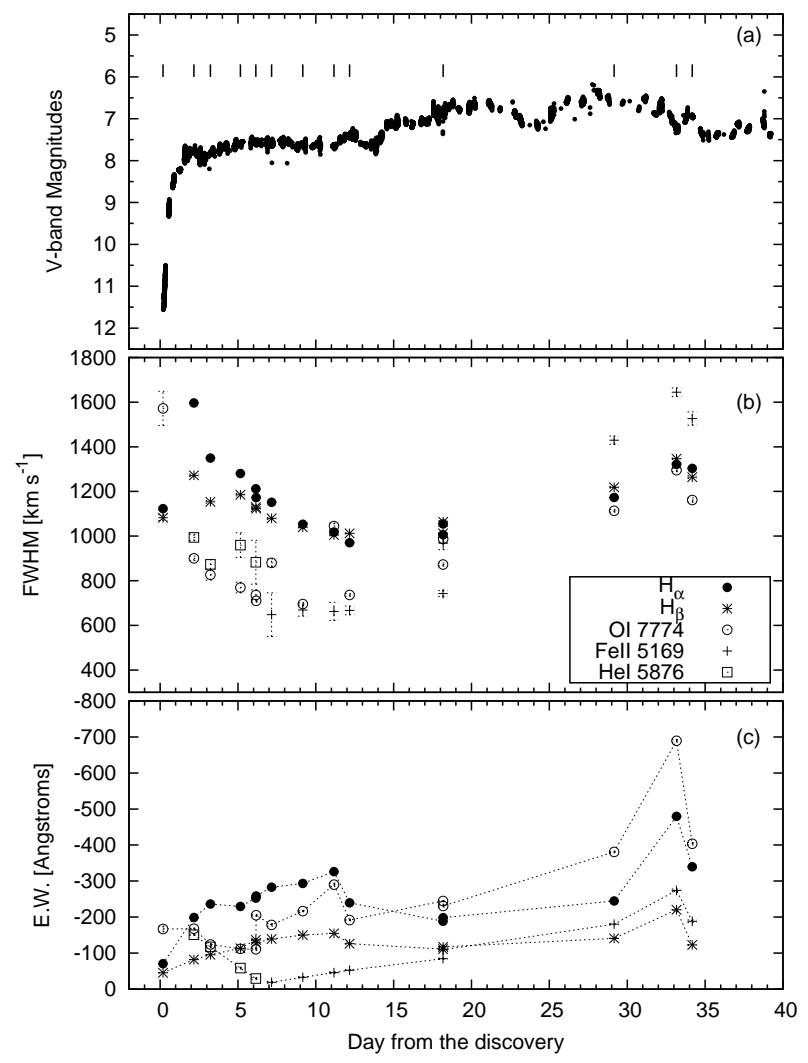

Figure 1: (a) The V-band light curve of the early phase of T Pyx 2011 quoted from AAVSO database. Short tics denote epochs of our observations. (b) The FWHMs of prominent emission lines. (c) The equivalent widths for the same lines in the panel (b).

\section{Results and Discussion}

\subsection{Wolf-Rayet like features in the initial rising phase on $t=0.19 \mathrm{~d}$}

Figure 2 shows our first spectrum on UT 2011 April $14.48(t=0.19 \mathrm{~d})$, just 4.4 hours after the discovery by M. Linnolt. The spectrum exhibits many highly excited emission lines, He II, C IV, N III, N IV, on this spectrum. The spectral features are reminiscent of WN type Wolf-Rayet (WR) stars (Crowther 2007 and references therein). We identified these emission lines with reference to spectral atlases of Wolf-Rayet stars (Hamann 1995, Conti 1990). The strong emission at C VI ( $\lambda 5802)$ is, however, observed in WC type rather than WN type.

In Wolf-Rayet stars, emission lines of highly excitation levels for $\mathrm{C}, \mathrm{N}$ or $\mathrm{O}$ are often observed as strong broaded emission lines $\left(\mathrm{FWHM}=800-2000 \mathrm{~km} \mathrm{~s}^{-1}\right.$ ). In generally, the stellar temperature for WR stars is around $5 \times 10^{4}-10^{5} \mathrm{~K}$, and WR winds are accelerated by line driven pressure (Crowther 2007).

In the ordinary picture of novae, during the initial rising phase, shells / a photosphere is expanding. Absorption components are expected to be detected for the Balmer and Fe II, O I, or He I transition. T Pyx showed, however, no significant P-Cygni profiles at the expanding velocities of highly excited emission lines (FWHM $\sim 1100 \mathrm{~km} \mathrm{~s}^{-1}$ ) on $t=0.19 \mathrm{~d}$. This implies that the envelope of WD would be optically thin on the moment, that is, the optically thick wind (Kato 1994) would be still weak on that time.

The T Pyx binary system has an orbital period of $1.83 \mathrm{~h}$ and a mass ratio of $0.2 \pm 0.03$ (Uthas et al. 2010). Such compact binary system has a dwarf companion, suggesting that the amount of surrounding gas supplied by the companion star of $\mathrm{T}$ Pyx is much less than the RS Oph-type RNe, which show highly excited emission by shock (e.g., [Fe VII]). Hence, the Wolf-Rayet like spectral features of T Pyx would be originated in the nova envelope, not by shocks with the circumbinary gas.

At this time, Wolf-Rayet like spectral features are reported for a few novae. In the PU Vul (1979), WN type features are discovered in optical and UV during its decline phase (Tomov et al. 1991, Sion et al. 1993). In the spectra of AG Peg, RR Tel and RT Ser, WR-like emission lines appeared after the visual maximum (Kenyon 1986, Tomov et al. 1991 and references therein). Our result is the first case of WR-like spectral features in the rising phase of novae.

\subsection{Developments after $t=2 \mathrm{~d}$}

Such WR-like spectral features disappeared in our spectra from $t=2 \mathrm{~d}$ as shown in Figure 3. No WR-like lines were reported after $t=0.8-1 \mathrm{~d}$ (Shore et al. 2011, Izzo et al., 2012, Surina et al. 2014). The brightness of T Pyx on $t=2 \mathrm{~d}$ was $V=8$, in the pre-maximum halt as shown in the panel (a) of Figure 1. On $t=2 \mathrm{~d}, \mathrm{H} \alpha, \mathrm{H} \beta$, O I, He I and N II have been strengthen, while emission lines of He II, C IV and N IV disappeared. This result indicates that the spectral type of T Pyx had changed from WR-like type to He/N type. The expanding velocity estimated from blue-shifted absorption minimum of P-Cygni profiles of $\mathrm{H} \beta$ is about $1400 \mathrm{~km} \mathrm{~s}^{-1}$. 
Optical Low Resolution Spectroscopic Observations of T Pyx during the Early Phase of 2011 Outburst

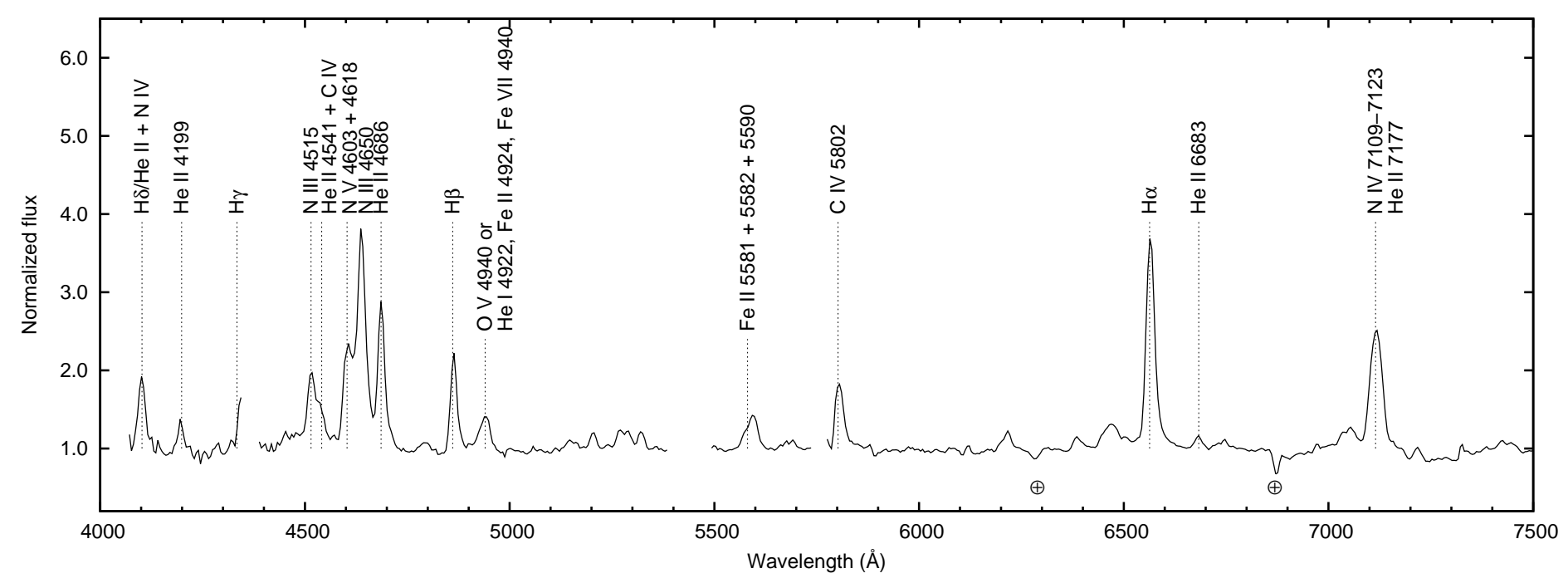

Figure 2: First spectrum of T Pyx on $t=0.19 \mathrm{~d}$. The highly excited emissions were detected likely to Wolf-Rayet stars. Cross circles denote positions of telluric absorption bands.

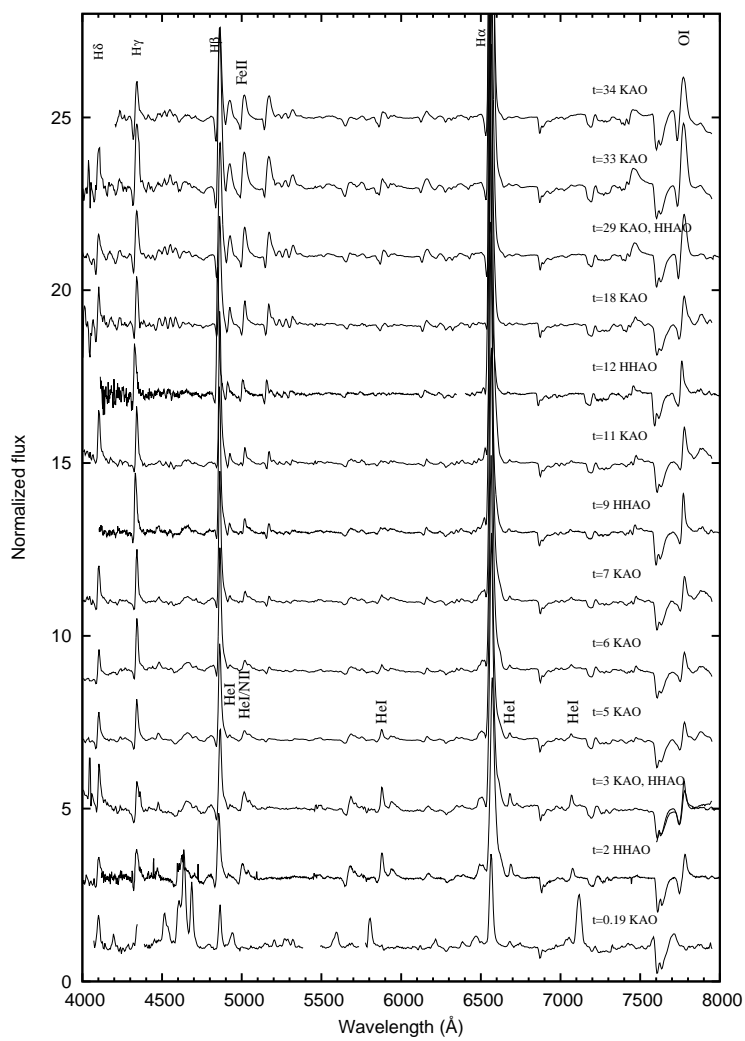

Figure 3: Low resolution spectra of $\mathrm{T}$ Pyx from $t=0.19-34 \mathrm{~d}$. Observations were carried out at Koyama Astronomical Observatory (KAO) and Higashi-Hiroshima Astronomical Observatory (HHAO). We identified the Balmer, He I, N II, Fe II multiplets, O I, Na I lines. The developments of FWHMs and equivalent widths of emission components of these lines are shown in the panel (b) and (c) of Figure 1, respectively.
The FWHM of $\mathrm{H} \alpha$ (and also $\mathrm{H} \beta$ ) measured in our spectra (the panel (b) of Figure 1) present a rapid increase from $1100 \mathrm{~km} \mathrm{~s}^{-1}$ to $1600 \mathrm{~km} \mathrm{~s}^{-1}$ during $t=0.19$ -2 d. Surina et al. (2014) reported faster velocity $\left(V_{e j}=4000 \mathrm{~km} \mathrm{~s}^{-1}\right)$ on $t=0.8 \mathrm{~d}$. After this event, the FWHM of $\mathrm{H} \alpha$ decreased gradually to $\sim 1000 \mathrm{~km} \mathrm{~s}^{-1}$ until $t=12 \mathrm{~d}$ in parallel with the increasing of their equivalent widths from - $70 \AA$ to $-320 \AA$, as shown in the panel(c) of Figure 1. FWHMs of other emission lines also showed similar trend, and their equivalent widths decreased to the minimum around $t=20 \mathrm{~d}$ (E.W. of $\mathrm{H} \alpha=-200 \AA)$. From $t=12 \mathrm{~d}$ to $t=34 \mathrm{~d}$, FWHMs of all lines increased again, from $\sim 1000 \mathrm{~km} \mathrm{~s}^{-1}$ to $\sim 1300 \mathrm{~km} \mathrm{~s}^{-1}$ for $\mathrm{H} \alpha, \mathrm{H} \beta$ and $\mathrm{O} \mathrm{I}(\lambda 7774) . \mathrm{H} \beta, \mathrm{N}$ II ( $\lambda 5679)$, O I ( $\lambda 7774)$ are accompanied with P-Cygni profiles clearly.

T Pyx showed a He/N-type spectra from $t=2 \mathrm{~d}$ to $t=6 \mathrm{~d}$, during pre-maximum halt. Decrease rates of equivalent widths of $\mathrm{He}$ I and N II are similar to the one observed in the Balmer lines at the same epoch. On the other hand, Fe II emission lines emerged aound $t=7 \mathrm{~d}$ as seen in the panel (c) in Figure 1. The equivalent widths of Fe II multiplets(42, 49) were growing gradually from $t=7 \mathrm{~d}$ to $t=34 \mathrm{~d}$. In this epoch, increasing trend of FWHM in Fe II and O I were also observed as seen in $\mathrm{H} \alpha$ and $\mathrm{H} \beta$.

On $t=34 \mathrm{~d}$, the equivalent width of Fe II and $\mathrm{O}$ I were slightly decreased due to the growth of continuum light in this epoch. These results indicate that the strong optically thick wind were gradually developing and Fe II type features replace a $\mathrm{He} / \mathrm{N}$ features around $t=7 \mathrm{~d}$, consisting with Surina et al. (2014). After the entering into the Fe II type, $\mathrm{H} \beta$, N II ( $\lambda 5679), \mathrm{O}$ 
I ( $\lambda 7774)$ are accompanied with clear P-Cygni profiles. These spectral developments are also reported and discussed in detail in Shore et al. (2011), Izzo et al. (2012), Imamura and Tanabe (2012), and Surina et al. (2014).

As pointed out by Imamura and Tanabe (2012), a similar hybrid behavior during the pre-maximum halt phase was also observed in only a slow developing nova, V5558 Sgr (Tanaka et al. 2011). T Pyx reveals intriguing spectral transition of spectral type of nova at more earlier stage than that observed in V5558 Sgr. The hybrid $(\mathrm{He} / \mathrm{N}$ - Fe II) transition would be a common behavior in slow rising novae. Very likely, the early observed WR-like features may be also a common evolution just after the explosion for slow rising novae.

Based on our results and combination with previous studies, we summarize that the development during the very early phase of the 2011 outburst of T Pyx is composed of three stages: (1) the WR-like stage, which shows short lived (duration $<1 \mathrm{~d}$ ) WR-like spectral line features, (2) the $\mathrm{He} / \mathrm{N}$ stage (several days), and (3) the Fe II-type stage.

\section{Acknowledgement}

We thank Taichi Kato (Kyoto University) for valuable comments on our observational data. This research was supported(, in part,) by a grant from the Hayakawa Satio Fund awarded by the Astronomical Society of Japan, and Optical \& Near-Infrared Astronomy Inter-University Cooperation Program, supported by the MEXT of Japan.

\section{References}

[1] Adams, W. S. and Joy, A. H. 1922, Publications of the American Astronomical Society, 4, 139

[2] Anupama, G. C., Evans, A., Bode, M. F., O'Brien, T. J. and Darnley, M. J. 2008, Astronomical Society of the Pacific Conference Series, 40, 31

[3] Catchpole, R. M. 1969, MNRAS, 142, 119 doi:10.1093/mnras/142.1.119

[4] Chomiuk, L., et al. 2011, The Astronomer's Telegram, 3318
[5] Conti, P. S. and Massey, P. and Vreux, J.-M. 1990, ApJ, 354, 359 doi:10.1086/168694

[6] Crowther, P. A. 2007, ARA\&A, 45, 177 doi:10.1146/annurev.astro.45.051806.110615

[7] Hamann, W.-R. and Koesterke, L. and Wessolowski, U. 1995, A\&AS, 113, 459

[8] Imamura, K. and Tanabe, K. 2012, PASJ, 64, L9

[9] Izzo, L., et al. 2012, MmSAI, 83, 830

[10] Joy, A. H. 1945, PASP, 57, 171 doi:10.1086/125711

[11] Kato, M. and Hachisu, I. 1994, ApJ, 437, 802 doi:10.1086/175041

[12] Kawabata, K. S., et al. 2008, SPIE, 7014, 4

[13] Nordsieck, K. H. and Shara, M. 2011, The Astronomer's Telegram, 3289, 1

[14] Kenyon, S. J. 1986, AJ, 91, 563

[15] Kuulkers, E, et al. 2011, The Astronomer's Telegram, 3285, 1

[16] Schaefer, B. E., Pagnotta, A. and Shara, M. M. 2010, ApJ, 708, 381

doi:10.1088/0004-637X/708/1/381

[17] Shinnaka, Y., et al. 2013, Icarus, 222, 734 doi:10.1016/j.icarus. 2012.08.001

[18] Shore, S. N., Augusteijn, T., Ederoclite, A. and Uthas, H. 2011, A\&A, 533, 8

[19] Sion, M. Edward et al. 1993, AJ, 106, 2118

[20] Surina, F., et al. 2014, AJ, 147, 107

[21] Tanaka, J., et al. 2011, PASJ, 63, 911

[22] Tomov, T., et al. 1991, MNRAS, 252, 31 doi:10.1093/mnras/252.1.31P

[23] Uthas, H. and Knigge, C. and Steeghs, D. 2010, MNRAS, 409, 237 doi:10.1111/j.1365-2966.2010.17046.x

[24] Waagan, E., et al. 2011, CBET, 2700, 1

[25] Williams, R. E. 1992, AJ, 104, 725 